\title{
Tunable white light generating nanocyrstal-hybridized LEDs
}

\author{
Hilmi Volkan Demir ${ }^{\mathrm{a}, \mathrm{b}, \mathrm{c}}$ and Sedat Nizamoglu ${ }^{\mathrm{a}, \mathrm{b}}$ \\ ${ }^{a}$ Department of Physics, ${ }^{b}$ Department of Electrical and Electronics Engineering, \\ ${ }^{c}$ Nanotechnology Research Center, \\ Bilkent University, Ankara, 06800, Turkey
}

\begin{abstract}
We hybridize CdSe/ZnS core-shell nanocrystals on InGaN/GaN near-UV/blue LEDs to generate light widely tunable across the visible spectral range and within the white region of the chromaticity diagram with adjustable tristimulus coordinates. We present the design, growth, fabrication, and characterization of these nanocrystalhybridized LEDs.
\end{abstract}

C2007 Optical Society of America

OCIS codes: (160.2540) Fluorescent and luminescent materials; (230.3670) Light-emitting diodes

To date white light-emitting diodes (WLEDs) have been implemented using different approaches including multi-chip WLEDs, multi-layer monolithic WLEDs, and color-conversion WLEDs, for example, using phosphor molecules coated on blue-emitting nitride LEDs. Among them, the color-conversion technique has been the most successful. However, the phosphors available for use in these devices present difficulties in controlling granule size and depositing films uniformly, which lead to undesired visible color variations [1]. Furthermore, phosphor photoemission properties are not easy to tune controllably. As an alternative to phosphor, we exploit nanocrystals (NCs) for use in color conversion to generate white light. To date we have introduced white light generation using $\mathrm{CdSe} / \mathrm{ZnS}$ core-shell nanocrystals of single, dual, trio, and quadruple combinations hybridized with blue and near-UV InGaN/GaN based LEDs. [2-4]. Also, a blue/green two-wavelength InGaN/GaN LED coated with a single type of red NC and a blue InGaN/GaN LED with a single type of yellow NC and a dual type of red and green NCs have been demonstrated [5-7].

In this conference, we present the hybridization of $\mathrm{CdSe} / \mathrm{ZnS}$ nanocrystals on InGaN/GaN LEDs to generate light that is widely tunable across the visible spectral range as shown in Fig. 1(a). Here we report the design, growth, fabrication, and characterization of these hybrid NC-LEDs. Fig. 1(b) shows an exemplary implementation of white light generation with such a nanocrystal-hybridized LED.

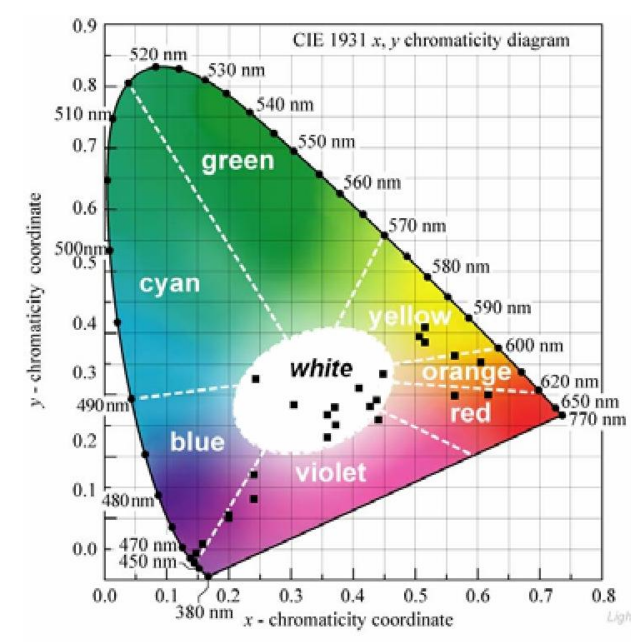

(a)

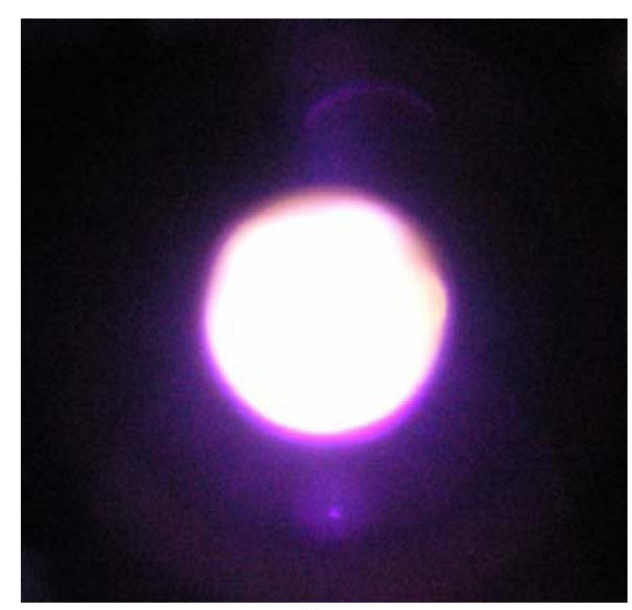

(b)

Figure 1. (a) ( $\mathrm{x}, \mathrm{y})$ tristimulus coordinates of our nanocrystal-hybridized LEDs on the C.I.E. (1931) chromaticity diagram and (b) photograph of white light generation from one of such hybrid NC-LEDs. 
We grow and fabricate $\mathrm{n}-\mathrm{UV}$ and blue $\mathrm{InGaN} / \mathrm{GaN}$ LEDs shown in Fig. 3 (a) and (c), respectively. Our n-UV LED has a peak electroluminescence at $383 \mathrm{~nm}$ and our blue LED, at 452 $\mathrm{nm}$, as shown in Fig. 3 (b) and (d) at different current injection levels at room temperature. We use 2-3 nm thick InGaN/GaN quantum well for the n-UV LED and 4-5 nm think well for the blue LED. We use four types of $\mathrm{CdSe} / \mathrm{ZnS}$ core-shell NCs shown in Fig. 3 (a)-(d) with their photoluminescence in the visible spectral range of cyan, green, yellow, and red and with their corresponding diameters ranging from $1.9 \mathrm{~nm}$ to $5.2 \mathrm{~nm}$. Our NCs exhibit photoluminescence peaks at $500 \mathrm{~nm}, 540 \mathrm{~nm}, 580 \mathrm{~nm}$, and $620 \mathrm{~nm}$, respectively, as characterized in Fig. 4 (e). Using different combinations of these nanocrystals hybridized on these LEDs, we conveniently obtain visible light generation widely tunable across the color diagram as demonstrated in Fig. 1(a).

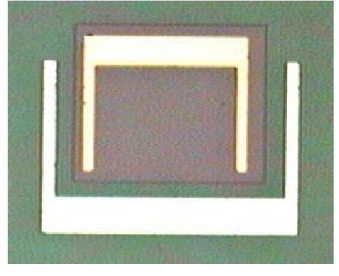

(a)

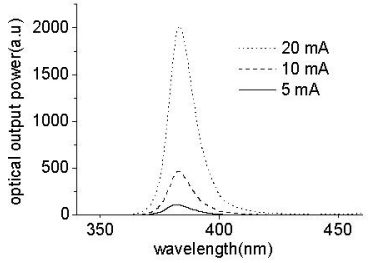

(b)

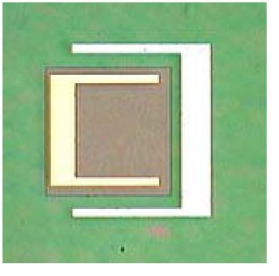

(c)

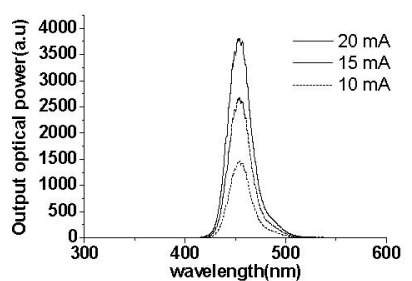

(d)

Figure 2. (a) micrograph of our near-UV InGaN/GaN LED and (b) its electroluminescence spectrum, and (c) micrograph of our blue InGaN/GaN LED and (d) its electroluminescence spectrum.

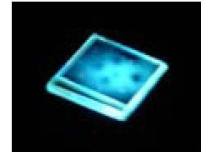

(a)

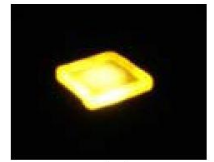

(c)

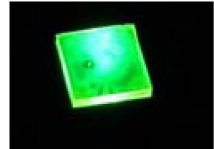

(b)

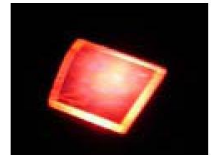

(d)

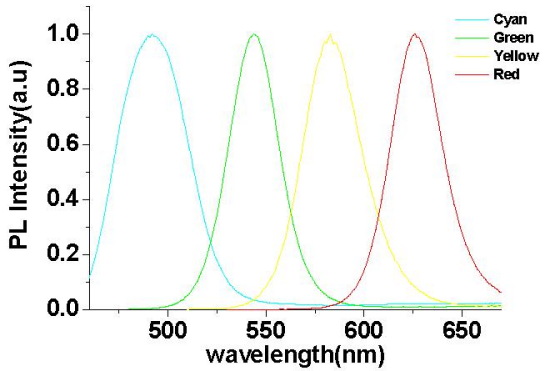

(e)

Figure. 3. Photoluminescence photographs of (a) cyan NC film, (b) green NC film, (c) yellow NC film, (d) red $\mathrm{NC}$ film, and (e) their corresponding photoluminescence spectra.

In conclusion, we hybridize cyan, green, yellow, and red $\mathrm{CdSe} / \mathrm{ZnS}$ core-shell nanocrystals on InGaN/GaN based blue/near-UV LED to generate light widely tunable across the visible spectral range and within the white region of the chromaticity diagram with adjustable tristimulus coordinates. These hybrid light sources hold promise for future lighting and display applications with their widely tunable color properties.

This work is supported by EU-PHOREMOST Network of Excellence 511616 and Marie Curie European Reintegration Grant MOON 021391 within the $6^{\text {th }}$ European Community Framework Program and TUBITAK under the Project Nos. 104E114, 106E020, 105E065, and 105E066. Authors acknowledge additional support from the Turkish Academy of Sciences and TUBITAK.

\section{References}

[1] Heliotis G, Gu E, Griffin C, Jeon C W, Stavrinou P N, Dawson M D and Bradley D D C 2006

J. Opt. A: Pure Appl. Opt. 8 445-449

[2] Nizamoglu S, Ozel T, Sari E and Demir H V 2007 Nanotechnology 18

[3] Nizamoglu S, Ozel T, Sari E, and Demir H V 2006 IEEE COMMAD Conference on Optoelectronic and Microelectronic Materials and Devices (Perth, Australia) WO-A5

[4] Nizamoglu S, and Demir H V 2007 Journal of Optics A: Pure and Applied Optics (in press).

[5] Chen H, Yeh D, Lu C, Huang C, Shiao W, Huang J, Yang C C, Liu I, and Su W 2006 IEEE Photon. Technol. Lett. 18 1430-1432

[6] Chen H, Hsu C, and Hong H 2006 IEEE Photon. Technol. Lett. 18 193-195

[7] Petruska M A, Koleske D D, Crawford M H, and Klimov V I 2006 Nano Lett. 6 1396-1400 